\title{
Hereditary Immunity and the Origin of Atherosclerosis
}

\author{
Sergey N. Rumyantsev, Vil K. Gerasimov, Jergy Grzezczuk, Roman A. Aron, \\ Nataly F. Avrova, Irina V. Belyakova, Ludmila V. Bobrakova, Kirill F. Dubrov, \\ Ludmila V. Perkus, Vladimir F. Pospelov, Nina M. Rogacheva, Nikolay P. Shabalov \\ Department of Evolutionary Immunology, Andent, Inc., Jersey City, USA \\ Email: rumyan1@yahoo.com
}

Received 23 January 2014; revised 23 February 2014; accepted 1 March 2014

Copyright (c) 2014 by authors and Scientific Research Publishing Inc.

This work is licensed under the Creative Commons Attribution International License (CC BY). http://creativecommons.org/licenses/by/4.0/

(c) (i) Open Access

\begin{abstract}
This paper tries to present new confirmation for the opinion about the infectious origin of atherosclerosis from the viewpoint of the current knowledge of hereditary immunity. The performed integrative investigation included the reinterpretation of known clinical and epidemiological observations, supported by immunological, molecular ecological, genetic, and genomic discoveries. The revealed body of firstly achieved information is compatible with the concept that infectious agents contribute the clue step to the development of atherosclerosis over its initial stage performed during the triggering of the arterial endothelial wall by the molecular agent of proposed infectious origin.
\end{abstract}

\section{Keywords}

Epidemic Spread; Heterozygosity; Homozygosity; Immune Mosaicism; Integrative Approach; Plaque Formation

\section{Introduction}

Atherosclerosis is a main disease that affects the cardiovascular system. It is accounted as the world's biggest killer. Since 1990, more people have died early worldwide from only one clinical form of atherosclerosis - the coronary artery disease - than from any other awesome disease. Cardiovascular diseases now represent more than $30 \%$ of all causes of deaths worldwide [1]. The disease can manifest in different clinical forms that affect the blood circulation: coronary artery disease, myocardial infarction, stroke, high blood pressure, or others. Any association with the aforementioned forms has been observed as well.

Atherogenesis is a chronic process affecting arterial blood vessels and is characterized by the formation of 
multiple atheromatous plaques within the arterial wall. They develop as a result of the accumulation of calcium, collagen, elastin, and fatty materials such as cholesterol and triglyceride in an arterial wall. These components of atheromatous plaques are composed of macrophages and other leucocytes nearest the lumen of the artery.

The buildup of an atheromatous plaque is a slow process, developed over a period of several years through a complex series of cellular events occurring within the arterial wall. One recent hypothesis suggests that, for unknown reasons, leukocytes, such as monocytes or basophils, begin to attack the endothelium of the artery lumen. The formation of atheromatous plaques is performed in the arterial tunica intima, a region of the vessel wall located between the endothelium and the tunica media.

Despite considerable theoretical, epidemiological, immunological, genomic, and pharmacological efforts, the epidemic of atherosclerosis continues to increase its toll on human life. The current efficacy of the disease prevention and treatment is very low. Even the etiology of atherosclerosis remains uncertain. The closely connected issues of the origin, initial causes, pathogenesis, and epidemic spread of atherosclerosis are not yet satisfactorily understood. There are several theories about how and why atherosclerosis develops.

The response-to-injury theory suggests that atherosclerosis develops as a result of the influence of lengthy injuring on the inner lining of the artery. A number of such injuring factors are considered by the apologists of this theory. The list of these factors include, first of all, the biochemical influence on the metabolism of the artery wall caused by a high concentration of cholesterol and low concentration of high-density lipoprotein in the blood. Second is the oxidative damage to the artery lining caused by free radicals formed during reactions between oxygen and low-density lipoprotein (LDL) cholesterol. Finally, some authors suppose that atherosclerosis develops as a response to an infection within the artery wall [2] [3].

The "response-to-endothelial injury" theory is the most widely accepted among researchers. However, nobody can confirm which ecological or physiological factor is responsible for the development of this "injury". Besides, this theory is especially impotent to interpret the stochastically spotted and individually peculiar disposition of atherosclerotic affections around the body. The epidemic spread of atherosclerosis around the world is also out of the scope of the "response-to-endothelial injury" theory. There is a gap in the understanding of events leading to the devastation, and as such, there are opportunities for novel ideas and concepts to explain the status quo and elaborate approaches to reform the situation dramatically.

The role of infection in the initiation of atherogenesis was also the subject of various trials to evidence it by discoveries and discussions. Multiple investigations have demonstrated that infectious agents evoke cellular and molecular changes supportive of such a role. Although a definitive proof of a causal role of infection contributing to it is lacking, some researchers believe that atherosclerosis may be caused by an infection of the vascular smooth muscle cells.

Chickens, for example, develop atherosclerosis when infected with the Marek disease herpesvirus [2] [4]. Herpesvirus infection of arterial smooth muscle cells has been shown to cause cholesteryl ester accumulation, which is associated with atherosclerosis [5]. Later, the findings were followed by the publications of similar associations between atherosclerosis and herpes simplex virus (HSV) types 1 and 2, Chlamydophila pneumoniae, Helicobacter pylori, and most recently, hepatitis A virus [6]. Cytomegalovirus infection is also associated with cardiovascular diseases [7]. The multiplicity of proposed infectious agents does not allow us to make certain conclusions and recommendations regarding the methods and means for effective prophylaxis and treatment of the disease.

In addition, although the injury to arterial vessel wall and the associated inflammatory response to the injury are now generally recognized as the essential components of atherogenesis, as many as $50 \%$ of patients with atherosclerosis lack currently identified noninfectious triggering risk factors (such as hypertension, smoking, hypercholesterolemia, and diabetes), an observation indicating that leading factors predisposing to atherosclerosis are as yet undetected.

There is a gap in the understanding of initial events leading to atherosclerosis, and as such, there are opportunities for novel ideas and concepts to explain the status quo and elaborate approaches to reform the situation dramatically. This paper tries to present new confirmation for the opinion about the infectious origin of atherosclerosis from the viewpoint of the current knowledge of hereditary immunity.

\section{Materials and Methods}

Functions of hereditary immunity in the genesis of atherosclerosis have been revealed by integrative interpreta- 
tion of relevant clinical and epidemiological observations, supported by immunological, molecular ecological, genetic, and genomic discoveries. This classical methodological approach [8] is widely used for the multidisciplinary discoveries of complex phenomena. At every of the named levels of the search, the main attention should be paid to the identification of the diversity of relevant traits, especially of those ones that are involved in the formation of the phenomenon of hereditary immune mosaicism [9] [10].

\section{Results and Discussion}

\subsection{Genetic Predilection to Atherosclerosis}

The development of atherosclerosis consists of two principally different events (Figure 1). The first event is performed by atherogenic triggers that injure the wall of endothelial cells and prepare them to the production of stuff needed to build the plaque. This is a clue event of atherogenesis. It opens the door for the complexity of secondary processes that are accomplished inside of the afflicted cell (Figure 1). However most of researchers devoted their attention to the genetics of secondary processes that form atherosclerotic plaques.

Atherosclerosis is an intra-arterial pathological process that induces inflammatory endothelial lesions with the appearance of lipid-containing foam cells. The earliest pathologic lesions of atherosclerosis are the fatty streaks that can be found in the arteries of many individuals by the age of 20 years. The fatty streaks are formed through the focal accumulation of serum lipoproteins within the intima of the vessel wall. The followed cholesterol metabolic pathways have a key role in atherosclerosis development [1]. They continue in the development and further progression of specific patched formations (plaques) on the innermost part of the arterial wall resulting in reduced or blocked blood flow.

However, there are many privileged individuals who are inherently resistant when challenged by triggering factors, thus preventing the secondary stage of atherogenesis. Knowledge of the resistant factors and their mechanisms of action would lead to a solution of the etiology [11]. This leads to the interest in hereditary immunity to atherosclerosis.

Recent investigations of monogenetic diseases, candidate genes, genetic polymorphisms, and susceptibility loci associated with atherosclerotic diseases have been identified in recent years. It has become obvious that genetic ground significantly influences susceptibility to atherosclerotic vascular diseases. The development of any case of atherosclerosis is now considered as a result of multifactorial triggering involving interactions of multiple genetic factors [11].

However, the known risk factors' features appear to be insufficient to explain the hereditary predilection to atherosclerosis. None of these risk factors, either alone or in couple, account for the entire contribution to the risk of atherosclerotic disease. In recent years, the interest in the identification of clue genetic risk factors to atherosclerosis continued to grow [11]. The search of clue factors is the most important goal of today's investigations.

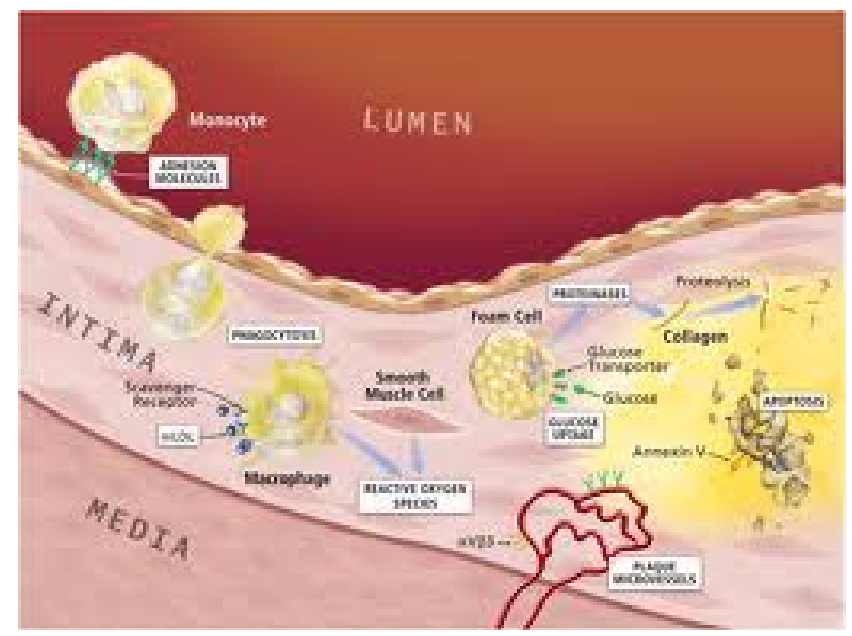

Figure 1. Schematic presentation of the development of atherosclerotic plaque in the wall of an artery (arterial disease). 


\subsection{Clinical Manifestations of Hereditary Immunity in Atherosclerosis}

The clinical entity of atherosclerosis results from a particularly complex disease process, with scope for a large degree of heterogeneity among patients who have apparently similar clinical presentations and, as a corollary, extensive intraindividual genetic heterogeneity. This disease is characteristic of especially variable interindividual and individual manifestations.

\subsubsection{Individual Locations and Sizes of Atherosclerotic Affections}

Individual manifestations and courses of atherosclerosis are highly variable. Many people with structurally advanced atherogenesis may have no symptoms and experience no functional impairment. On the other hand, initially asymptomatic patients may be subjected to sudden cardiac or aortal death, whereas in many other people, the disease presents for a long time with clinically important symptoms and signs of relevant chronic diseases (coronary heart disease, high blood pressure, renal deficiency, or other local dysfunction of blood circulation), or they may suffer from various consequences of former myocardial infarction or stroke. Initially thought to be a chronic, slowly progressive, degenerative disease, it is now apparent that atherosclerosis is a disease with periods of activity and quiescence.

Although named a systemic disease, atherosclerosis manifests in a local manner and affects stochastically different organs and systems in different patients (Figure 2) for reasons that are accounted as unclear. Being specific elements of atherogenesis, the plaques may vary in location, quantity, and size. Any individual case of atherosclerosis begins when some initial injury of arterial intima induces the fatty molecular material to deposit at the injured place in the form of plaques, resulting in the focal narrowing of the arterial lumen and the eventual impairment of blood flow.

Plaques can be either single or multiple depending on the presence on the arterial endothelium of the quantity of places not immune to the relevant triggering agent. The nonimmune places are usually localized stochastically on the wall of either one or some of the arterial vessels. The cases of total affliction of large endothelial surfaces or many vessels many are extraordinarily seldom, which indicate the presence and heterozygosity of hereditary immunity to atherosclerosis among most of currently living people.

Coronary (heart) artery atherosclerosis leads to coronary artery disease, that is, to chest pain, heart attack, myocardial infarction, and sudden death. Thoracic aorta atherosclerosis leads to aortic aneurysm. Atheroma in arm or leg arteries may cause occlusive disease of relevant peripheral artery. In carotid arteries of the brain, the atherosclerosis leads to sudden dizziness, weakness, loss of hearing or speech, and blindness. Cerebral artery atherosclerosis may lead to a stroke on one side of the body. Hepatic artery atherosclerosis leads to insufficiency of the relevant lobe of the liver. Affection of the pancreatic artery leads to insufficiency of the pancreas.

Abdominal aorta atherosclerosis leads to abdominal aorta aneurysm. The location of plaques in a mesenteric artery can induce ischemic bowel disease. Atherosclerosis of a renal artery can lead to insufficiency of the relevant kidney, for instance, high blood pressure resistant to treatment. Peripheral artery atherosclerosis is caused by plaque buildup in the arteries that supply the extremities of the body (such as the hands and feet) with blood. In the femoral artery of the legs, the atherosclerosis leads to cramping and fatigue in the calves of the legs when walking and, finally, to the necrosis of the legs or fingers.

\subsubsection{Variations in the Course and Severity of Atherosclerosis}

Early lesions of the endothelium appear as fatty streaks. These later develop into atherosclerotic plaques (Figure 1), which consists of a lipid core and an overlying fibrous cap. Atherosclerotic plaques can either remain silent for a long time or progressively narrow the lumen, which restricts flow (e.g., producing angina pectoris). Rupture or erosion of a plaque can precipitate an acute occlusion owing to the formation of a thrombus or blood clot. In some cases, the progression of the plaques can lead to rupture of the arterial wall and appropriate bleeding.

Plaque buildup in the vessels that supply an organ with blood may cause dysfunctions of the relevant organ. Symptoms of any atherosclerotic affection depend on the size of the affection's location as well as on the danger of the focal bloodstream blockage (Figure 2). The most clinically important manifestations of the disease are focal and tend to occur at individually located sites.

The symptoms of atherosclerosis differ at first depending on the location of plaques (Figure 2) as well as on both their size and the grade of development. Any local affection may exist either alone or in any association with an affection of any other locations. In some patients, the intensity of the affection and its progression is rather high, whereas in others, the manifestations are weak. The local character of the affection is the principal 


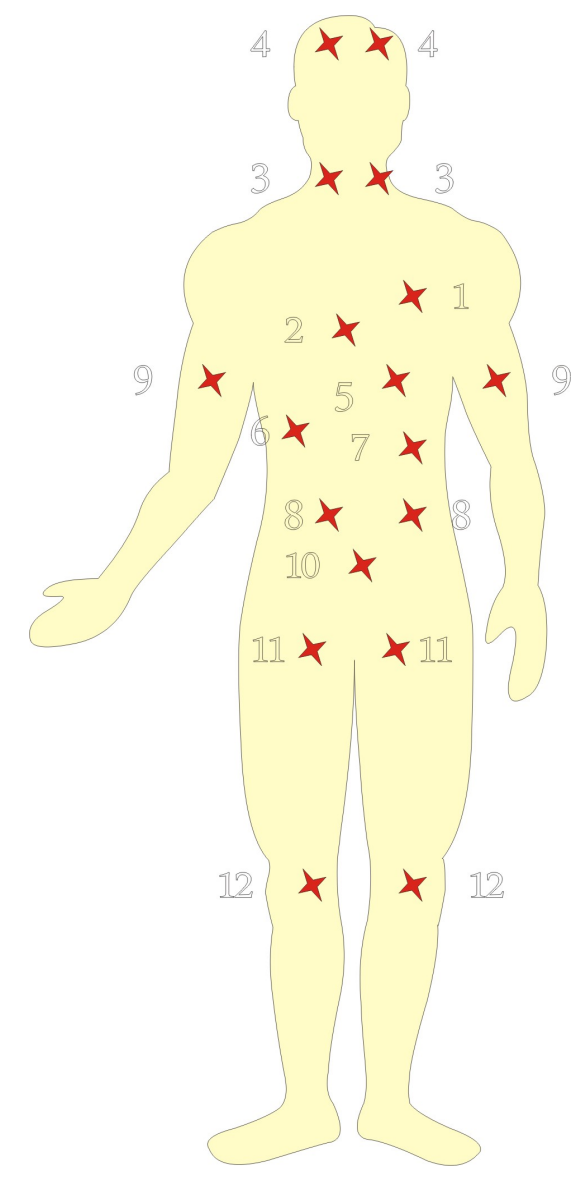

Figure 2. Most dangerous locations of atherosclerotic affections: 1) aorta (aortic aneurysm), 2) coronary artery (coronary arterial disease), 3) internal carotids (stroke), 4) cerebral arteries (stroke), 5) abdominal aorta (aortic aneurysm), 6) hepatic artery (insufficiency of liver), 7) pancreatic artery (insufficiency of the pancreas), 8) renal arteries (kidney ischemia, a silent killer), 9) brachial arteries (peripheral arterial disease), 10) abdominal aorta (abdominal aortic aneurysm), 11) femoral arteries (peripheral arterial disease), 12) tibial arteries (peripheral arterial disease).

feature of clinical manifestations in this kind of pathology.

The progression of the plaques leads to the narrowing of the affected arteries and relevant local limitation of blood flow. Atherosclerosis usually does not cause symptoms until it severely narrows an artery or totally blocks an artery. Usually, symptoms begin when there is at least a 75\% blockage in the locality of the affected artery. As a plaque encroaches on the luminal area, blood flow becomes limited, and flow cannot increase on demand.

Any artery in the body as well as any part of the artery can be involved, but only in severe local narrowing or obstruction of some arteries, those that supply more critically important organs-first of all, heart, brain, and kidney - is atherosclerosis revealed in its life-threatening form (Figure 2). Plaques may also build up in more than one place in the artery. According to the location and degree of specific affections, this can result in relevant local ischemic accidents that manifest appropriate clinical syndromes and outcomes [1]. Moreover, the unequal affection of different parts of an organism's circulatory system is uneven as well [12]. 


\subsection{Origin of Intraindividual Immune Diversity}

Although the identification of susceptibility genes for these common, complex diseases is a vital goal, the real progress in unearthing novel genes has been slow. The optimistic predictions of the impact of genetics on the diagnosis and prevention of atherosclerosis [13] cannot be realized because it is impossible to imagine the existence of genes responsible for any individual localization, size, or intensity of the development of atherosclerotic affection. Classic genetics cannot explain why one person reveals only one asymptomatic small plaque on the wall of a peripheral artery, whereas another person suffers from that unable to provide very important organs with blood. In other words, hereditary genetic factors are able to determine while some small parts of the arterial wall are receptive to atherogenic triggering, whereas far more extensive neighboring parts of the same vessel are not injured.

Hereditary immunity is a result of natural selection performed in the population by infectious epidemic processes. Most members of the population become inherently immune, whereas some heterozygotes possess both immune and susceptible loci in their bodies (Figure 3(c)). The quantity of susceptible loci may be very small, which is observed in the cases of solitary atherosclerotic plaques.

\subsection{Manifestations of Hereditary Immunity in Epidemiological Observations}

\subsubsection{Population Diversity in the Prevalence of Atherosclerosis}

Thus, it becomes obvious that humans, that is, different parts of their individual aortic circulatory systems, reveal extraordinary genetic diversity in their predisposition to atherosclerosis. Affected people have many differences in the manifestations of their atherosclerosis, especially in its location, size, and the grade of expression. Individuals of an observed human population can be conveniently divided into at least four principal categories, as follows:

1) The totally unaffected (homozygous) organisms that have no atherosclerotic plaques. Those people formed substantial quotes of some modern populations.

2) Mildly affected (heterozygous) organisms in which few plaques have appeared, whereas other areas of the body arterial vessels are left unaffected.

3) Mostly serious form of the disease with intermediate grade of atherosclerosis formed by location of solitary plaques in the artery of especially important organs.

4) Extraordinarily rare but exceptionally dangerous disease with dissemination of multiple atherosclerotic lesions in many parts of the arterial basin.

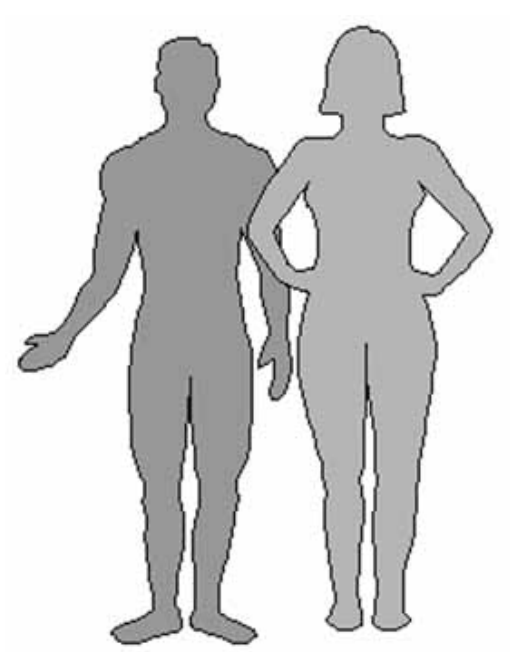

(a)

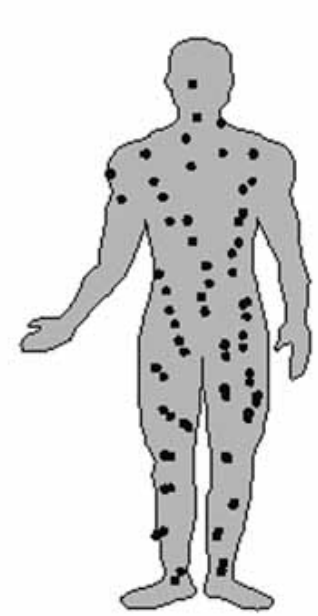

(c)

Figure 3. Origin of intraindividual immune diversity: (a) father, a homozygous man with high susceptibility to atherosclerosistriggering agent; (b) mother, a homozygous woman hereditarily immune to the agent; (c) their hybrid (heterozygous) offspring of intermediate immunity [14]. 
In Finland, 835 people of every 100,000 of the population die of heart disease. China, with 81 per 100,000 people affected, has one of the lowest. Among women, the highest rate in the world is seen in Scotland, with the lowest in Catalonia in Spain [15]. In contrast, the frequency of the disease among "nonindustrialized" populations of the Far East is significantly lower than that in the West. Atherosclerosis is also rare among the native populations of the African continent with a traditionally rural and agrarian way of life.

This kind of pathology is more characteristic of the mixed populations. Recently, its prevalence in developing countries has rapidly increased as well. The epidemic of cardiovascular diseases has taken on a global dimension. Women are more likely to die than men-55\% of the people who died from cardiovascular disease are women.

The future identification of specific susceptibility genes will add to our knowledge of the molecular pathophysiology of atherosclerosis [1], but it will not explain the phenomenal diversity in the location and size of atherosclerotic affections, which is the main factor that determines the location, the course, the severity, and the exit of any case of disease. It will not refine the identification of high-risk individuals and suggest areas of research for drug discovery. Escape from atherosclerosis can be achieved on the basis of its prophylaxis, which should be elaborated by medical genetics.

\subsubsection{Ethnic and Racial Disparity in Predisposition to Atherosclerosis}

Studies of atherogenic vascular pathology have shown substantial racial differences in the predilection to atherosclerosis. Among Caucasians, the pathology is likely to be in a proximal location such as the bifurcation of the carotid arteries in the neck. This type of lesion is accessible to surgical correction. Among the Orientals, the vascular degeneration is more likely to occur within the brain. The Orientals are also more likely to develop small vessel diseases that may cause different types of stroke, including the hemorrhagic one [5]. Orientals are generally known to have more intracranial vascular stenosis and a lower incidence of carotid stenosis than Caucasians [16].

In addition, some observations indicate allelic heterogeneity for genetic susceptibility to atherosclerosis [1]. The Icelandic HapA risk haplotype was associated with increased neutrophil release of leukotriene B4 (LTB4), which is synthesized from LTA4, indicating that the ALOX5AP variants might be proinflammatory. LTB4 activates monocytes, which migrate through the arterial endothelium and differentiate into macrophages that take up lipid to become foam cells, a key early event in atheroma development. Blockade of the LTB4 receptor has been shown to reduce the amount of lipid accumulation, monocyte infiltration, and lesion size in Ldlr-/- or ApoE-/(apolipoprotein E) knockout mouse models of atherosclerosis (which are susceptible to atherosclerosis owing to dyslipidemia). There is still a large gap in our knowledge of the genes that are involved in susceptibility for most patients with multifactorial coronary artery disease [1]. But the most gap is formed by phenomenal diversity in the location and size of atherosclerotic affections.

\subsubsection{Global Variations in the Prevalence of Atherosclerosis}

Atherosclerosis is the most common cause of death not everywhere in the world but among some populations of "industrialized" countries, especially in the United States, England, Scotland, Finland, Scandinavia, and Russia. As health improvements and industrialization come to developing countries, they also start to adopt what are considered "Western habits," such as smoking, less physical activity, and a diet rich in fatty fast foods. Atherosclerosis is becoming more prevalent in the Western world and is beginning to become prevalent in countries where the populations are adopting Western lifestyles including xenogamous admixture [18].

\subsection{Conclusion}

During the last two decades of XX century, researchers have proposed bacterial and viral triggers for some diseases that have been accounted as noninfectious before [17]. Many bacteria and viruses have been linked to formerly "noninfectious" diseases. However, despite the growing evidence, the role of infectious agents in a wide range of diseases is still contested. For decades, scientists have unsuccessfully attempted to link infection with cancer, atherosclerotic cardiovascular disease, obesity, and so on. The direct triggering role of microbial agents in the named diseases continues to be very controversial in contrast to the indirect one [18]. This group of diseases continues to be considered noninfectious. The performed investigation on the origin of atherosclerosis from the viewpoint of hereditary immunity reveals a body of information compatible with the concept that infectious agents contribute the clue step to the development of atherosclerosis over its initial stage. 


\section{References}

[1] Watkins, H. and Farrall, M. (2006) Genetic Susceptibility to Coronary Artery Disease: From Promise to Progress. Nature Reviews Genetics, 7, 163-173. http://dx.doi.org/10.1038/nrg1805

[2] Fabricant, C.G. (1984) Herpesvirus-Induced Atherosclerosis. Springer Verlag, New York.

[3] Hajjar, D.P., Fabricant, C.G., Minick, C.R. and Fabricant, J. (1986) Virus-Induced Atherosclerosis. Herpesvirus Infection Alters Aortic Cholesterol Metabolism and Accumulation. American Journal of Pathology, 122, 62-70.

[4] Fabricant, C.G. and Fabricant, J. (1999) Atherosclerosis Induced by Infection with Marek’s Disease Herpesvirus in Chickens. American Heart Journal, 138, S465-S468. http://dx.doi.org/10.1016/S0002-8703(99)70276-0

[5] Hsu, C.Y. (1994) Recent Advances in the Development of Therapeutics to Reduce Brain Damage after Stroke. 7th International Conference on Health Problems Related to the Chinese. The Chinese American Medical Society, New York.

[6] Zhu, J., Quyyumi, A.A., Norman, J.E., Costello, R., Csako, G. and Epstein, S.E. (2000) The Possible Role of Hepatitis A virus in the Pathogenesis of Atherosclerosis. The Journal of Infectious Diseases, 182, 1583-1587. http://dx.doi.org/10.1086/317613

[7] Cheng, J., Ke, Q., Jin, Z., Wang, H., Kocher, O., Morgan, J.P. and Crumpacker, C.S. (2009) Cytomegalovirus Infection Causes an Increase of Arterial Blood Pressure. PLOS Pathogens, v. 5.

[8] Darwin, C. (1888) The Descent of Man and Selection in Relation to Sex. John Murray, London.

[9] Rumyantsev, S.N. (2008) Phenomenon of Immune Mosaicism. Hereditary Immunity: Fundamental Principles and Exploitation in Life Study and Health Care. Nova Science Publishers, New York, 81-102.

[10] Rumyantsev, S.N. (2010) Bioecology of Pleistocenic Spurt in Anthropogenesis. International Journal of Integrative Biology, 10, 14-21.

[11] Weber, C. and Noels, H. (2011) Atherosclerosis: Current Pathogenesis and Therapeutic Options. Nature Medicine, 17, 1410-1422. http://dx.doi.org/10.1038/nm.2538

[12] Sing, S.F. and Moll, P.P. (1990) Genetics of Atherosclerosis. 171-187.

[13] Flegal, K.M., Carroll, M.D., Kuczmarski, R.J. and Johnson, C.L. (1998) Overweight and Obesity in the United States: Prevalence and Trends, 1960-1994. International Journal of Obesity, 22, 39-47. http://dx.doi.org/10.1038/sj.ijo.0800541

[14] Rumyantsev, S.N. (2003) The Intra-Individual Diversity in Senescence. Biogerontology, 4, 171-178. http://dx.doi.org/10.1023/A:1024137418419

[15] Kathryn Senior Ph.D. Facts and Figures: Heart Disease Worldwide. Cardiac matters.co.uk, 2013.

[16] Chu, J. (1994) Epidemiology of Cerebrovascular Disease among Chinese-Canadians. 7th International Conference on Health Problems Related to the Chinese. The Chinese American Medical Society, New York.

[17] Carbone, K.M., Luftig, R.B. and Buckley, M.R. (2005) Microbial Triggers of Chronic Human Illness. American Academy of Microbiology, Washington DC.

[18] Rumyantsev, S.N. (2006) Obesity: A Reckoning Both for Genetic Immunity to Infection and Xenogamy. Medical Hypothesis, 66, 535-540. http://dx.doi.org/10.1016/j.mehy.2005.09.014 